\title{
Evidencia de transmisión autosómica dominante a través de la línea materna en un caso de discinesia ciliar primaria
}

\author{
Álvarez González $\mathrm{J}^{1,2,3}$, Busto Castañón $\mathrm{L}^{4}$, Nistal Serrano $\mathrm{M}^{5,6}$. \\ ${ }^{1}$ Centro de Infertilidad Masculina ANDROGEN, La Coruña, ${ }^{2}$ Harvard Medical School, Boston. ${ }^{3}$ Instituto \\ Marqués. Barcelona. ${ }^{4}$ Clínica Urológica Dr. Busto Castañon, La Coruña. ${ }^{5}$ Hospital Universitario La Paz, \\ Madrid, ${ }^{6}$ Universidad Autónoma de Madrid.
}

Actas Urol Esp. 2006;30(7):728-730

\section{RESUMEN}

\section{EVIDENCIA DE TRANSMISIÓN AUTOSÓMICA DOMINANTE A TRAVÉS DE LA LÍNEA MATERNA EN UN CASO DE DISCINESIA CILIAR PRIMARIA}

Se presenta evidencia de un caso atípico de discinesia ciliar primaria en el que la transmisión genética, en lugar de la clásica autosómica recesiva, parece ser de tipo autosómico dominante transmitida a través de la línea materna. Se trata de un caso de dos hermanos de 29 y 30 años de edad casados y sin hijos, con historia de infertilidad, sinusitis frecuentes e infecciones respiratorias recurrentes. Madre y hermana presentan broncopatía crónica no filiada. Padre sano sin afectación pulmonar o sinusal. En el examen físico, en ambos hermanos, la hermana, y la madre, sólo destaca la presencia de roncus en la auscultación pulmonar. Bioquímica ordinaria normal, incluyendo pruebas de función pulmonar, hepática y renal. El estudio ultraestructural del flagelo espermático por microscopía electrónica indica que los dos hermanos tienen la misma anomalía. En la mayoría de las secciones transversales se encuentra ausencia de ambos brazos de dineína. El filamento de nexina está presente, así como las fibras radiales y el par central. En espermatozoides aislados, coincidiendo con la ausencia de los brazos de dineína, hay también pérdida del par central. No se encontraron anomalías en la vaina fibrosa, ni pérdida de las fibras densas. La pieza intermedia en aproximadamente el 50\% de los espermatozoides mostraba una disminución en el número de mitocondrias y de mitocondrias extras no alineadas. Otros hallazgos fueron la presencia de microtúbulos extra periféricos al axonema.

Palabras clave: Discinesia ciliar. Inmotilidad. Síndrome del cilio inmóvil. Infertilidad.

\section{ABSTRACT}

\section{EVIDENCE FOR AUTOSOMAL DOMINANT INHERITANCE THROUGH THE MATERNAL LINE IN A} CASE OF PRIMARY CILIARY DISKINESIA.

An atypical case of primary ciliary dyskinesia is presented in which the inheritance, rather than the classical autosomal recessive, appears to be transmitted as an autosomal dominant trait through the maternal line. The case involves two brothers of 29 and 30 years of age, married without children, with a history of infertility, frequent episodes of sinusitis, and recurrent pulmonary infections. Their mother and sister have chronic bronchopathy of unknown etiology. Their father is healthy without pulmonary problems or sinusitis. At physical exam, both brothers, sister and mother presented with bronchial rhonchi at lung auscultation. Blood analysis and pulmonary function, liver and renal tests were all normal. The ultraestructual study of the sperm flagellum by electron microscopy revealed that both brothers have the same anomaly. Namely, in the majority of the cross-sections, both dynein arms are missing. The nexin filament was present, as well as the radial spokes and the central pair of microtubules. In some sperm, besides the absence of dynein arms, there was also absence of the central pair of microtubules. Neither anomalies of the fibrous sheath nor of the dense fibers were found. In approximately $50 \%$ of the spermatozoa, the midpiece had a decreased number of mitochondria and extra non- aligned mitochondria. Other findings included extra peripheral microtubules in the axoneme. 
$\mathrm{L}^{\mathrm{a}}$ a discinesia ciliar primaria (DCP) es una enfermedad autosómica recesiva, con una incidencia de aproximadamente 1 caso de cada 16.000 habitantes. De estos, la mitad presentan la tríada de Kartagener (sinusitis, bronquiectasias y situs inversus). La DCP parece ser un grupo heterogéneo de trastornos con diferentes y variadas alteraciones ultraestructurales de los cilios y axonema del espermatozoide, siendo la más frecuente la deficiencia de los brazos de dineina y del par de microtúbulos central ${ }^{1-5}$. En pacientes con DCP familiar se ha identificado una alteración en el cromosoma 5p15-p14, y más concretamente en el gen DNA H5 ${ }^{6}$.

La presentación clínica de la DCP incluye el síndrome de Kartagener, sinusitis crónica, otitis, enfermedad pulmonar crónica o infertilidad debido a la inmovilidad total de los espermatozoides. La ausencia o ineficacia de la actividad ciliar conlleva a infecciones pulmonares y sinusales crónicas, afectándose además el desplazamiento visceral durante el período embrionario, responsable del situs inversus ${ }^{7}$.

\section{CASO CLÍNICO}

Hermanos de 29 y 30 años de edad casados y sin hijos, con historia de infertilidad y sinusitis frecuentes e infecciones respiratorias de repetición desde hace muchos años, aunque niegan haberlas padecido en la infancia. Madre y hermana presentan broncopatía crónica no filiada. Padre sano sin afectación pulmonar o sinusal. En el examen físico de ambos hermanos, hermana, y de la madre, sólo destaca la presencia de roncus en la auscultación pulmonar. No se pudieron obtener antecedentes clínicos de la ascendencia tanto paterna como materna. Bioquímica ordinaria normal, incluyendo pruebas de función hepática y renal. Alfa-1-anti-tripsina normal. Proteinograma e inmunoglobulinas normales. Hormonas tiroideas normales. Marcadores del virus B de la hepatitis y del virus de la inmunodeficiencia humana negativos. Anticuerpos antinucleares, pruebas reumáticas, fracción C3 y C4 negativos. Gasometría arterial basal y bicarbonato normal. Espirometría normal. Saturación $\mathrm{Hb}$ normal. Radiografía de tórax normal. Tanto los hermanos como la hermana y la madre rehusaron hacer biopsia de mucosa respiratoria para estudio ultraestructural de los cilios. El análisis de semen indica astenozoospermia total en 3 seminogramas diferentes realizados a ambos hermanos, con una concentración espermática media de 23 millones $/ \mathrm{ml}$, un porcentaje de formas normales por criterios de Kruger del 6\% y una viabilidad del 95\%. El estudio ultraestructural del flagelo espermático por microscopía electrónica indica que los dos hermanos tienen la misma anomalía. En la mayoría de las secciones transversales se encuentra ausencia de ambos brazos de dineína (Fig. 1). El filamento de nexina está presente, así como las fibras radiales y el par central. En espermatozoides aislados, coincidiendo con la ausencia de los brazos de dineína, hay también pérdida del par central. No se encontró anomalía en la vaina fibrosa, ni pérdida de fibras densas. La pieza intermedia en la mitad aproximadamente de los casos tenía disminución del número de mitocondrias y mitocondrias extras no alineadas (Fig. 2). Otros datos añadidos fueron la presencia de microtúbulos extra periféricos al axonema.

\section{DISCUSION}

La transmisión genética de la DCP es normalmente autosómica recesiva. El hecho de que ambos hermanos presenten la misma anomalía y que su hermana y su madre tengan afectación pulmonar crónica y sinusitis, sugiere que quizás podría tratarse de una transmisión atípica dominante transmitida a través de la línea materna.

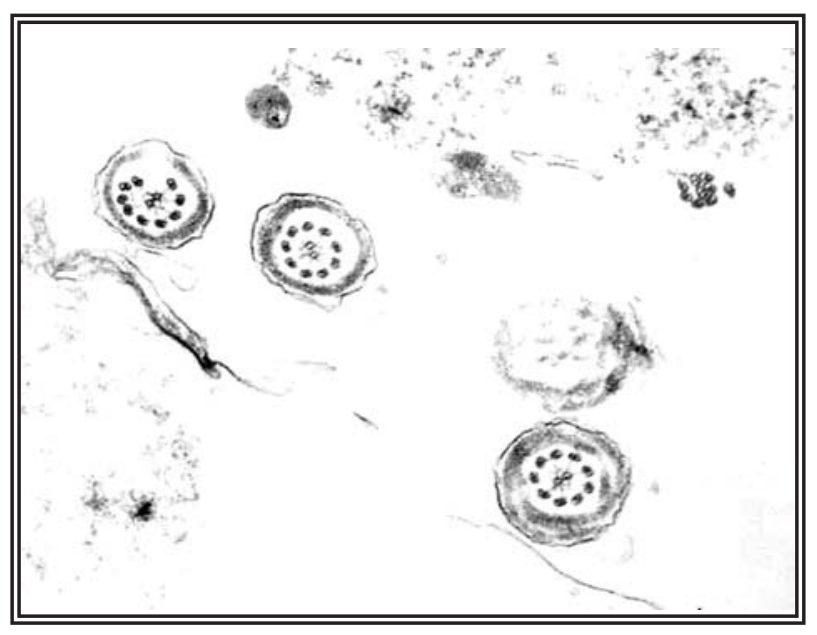

FIGURA 1. Imagen ultraestructural de microscopia electrónica mostrando ausencia de los brazos de dineína (X 48.500). 


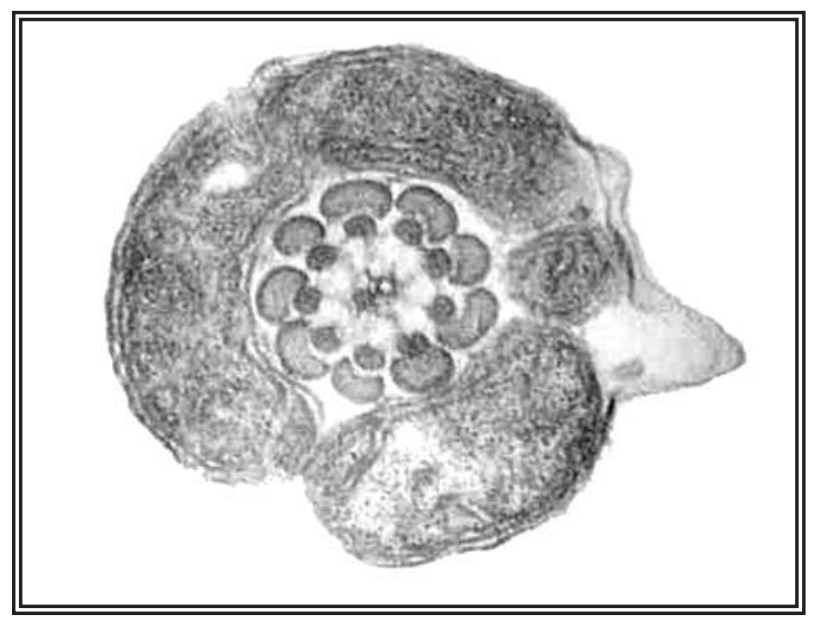

FIGURA 2. Imagen ultraestructural de microscopia electrónica en la que se puede observar que existen anomalias a nivel de la pieza intermedia relacionadas con la alineación y el número de mitocondrias ( $X$ 262.800).

Narayan et al., describieron un caso similar en el que la madre y sus 5 hijos, nacidos de tres padres diferentes, estaban afectados de discinesia ciliar primaria. Ninguno de los tres matrimonios de la madre fueron consanguíneos ${ }^{8}$. Este hallazgo sugiere que podría tratarse de una transmisión autosómica dominante o bien de una transmisión ligada al cromosoma $\mathrm{X}$.

En el caso que nos ocupa, a diferencia del caso reportado por Narayan et al., además de la madre y de los dos hermanos, la hija también parece estar afectada de discinesia ciliar primaria. Esto descartaría una transmisión ligada al X y sugeriría una transmisión autosómica dominante transmitida a través de la línea materna. $\mathrm{Si}$ la transmisión fuese ligada al sexo, no estaría afectada la hermana. Sin embargo, dado que no se realizó un estudio ultraestructural de los cilios de la mucosa respiratoria ni en la madre ni en la hija, no se puede afirmar que estas estuviesen afectadas de discinesia ciliar primaria.

\section{CONCLUSIONES}

Los resultados de este estudio sugieren la posibilidad de transmisión autosómica dominan- te ligada a la línea materna en casos de DCP. Por lo tanto, pacientes afectados de DCP deberian ser debidamente informados por el genetista sobre esta posibilidad, especialmente cuando contemplen tener descendencia utilizando técnicas de in vitro como ICSI.

Agradecimientos: Nos gustaría expresar nuestro agradecimiento al Dr. Hector Chemes por su revisión crítica del manuscrito.

\section{REFERENCIAS}

1. Afzelius BA. A human syndrome caused by immotile cilia. Science 1976;193(4250):317-319.

2. Ishii S, Minato K, Hagiwara H, Yonezu M, Shimomura K, Iizuka K, et al. A possible mechanism of primary ciliary dyskinesia: a case of a segmental defect in ciliary microtubules. Intern Med, 1999;38(7):602-606.

3. Escudier E, Escalier D, Pinchon MC, Boucherat M, Bernaudin JF, Fleury-Feith. Dissimilar expression of axonemal anomalies in respiratory cilia and sperm flagella in infertile men. Am Rev Resp Dis. 1990;142(3):674-679.

4. Torikata C, Kijimoto C, Koto M. Ultrastructure of respiratory cilia of WIC-Hyd male rats. An animal model for human immotile cilia syndrome. Am J Pathol, 1991;138(2):341-347.

5. Chemes EH and Rawe YV. Sperm pathology: a step beyond descriptive morphology. Origin, characterization and fertility potential of abnormal sperm phenotypes in infertile men. Hum Reprod Update, 2003; 9(5):405-428. Review.

6. Olbrich H, Haffner K, Kispert A, Volkel A, Volz A, Sasmaz G, Reinhardt R, Hennig S, Lehrach H, Konietzko N, Zariwala M, Noone PG, Knowles M, Mitchison HM, Meeks M, Chung EMK, Hildebrandt F, Sudbrak R, Omran H. Mutations in DNAH5 cause primary ciliary dyskinesia and randomization of left-right asymmetry. Nature Genet, 2002; 30: 143-144.

7. Robbins CK. El aparato respiratorio. Patología Estructural y Funcional. Tomo II ( $4^{\mathrm{a}} \mathrm{ed}$.). Inter Americana McGraw Hill. Madrid 1990. p. 819-820.

8. Narayan D, Krishnan SN, Upender M, Ravikumar TS, Mahoney MJ, Dolan TF Jr, Teebi AS, Haddad GG. Unusual inheritance of primary ciliary dyskinesia (Kartagener's syndrome). J. Med. Genet, 1994;31(6):493-496.

Dr. J.G. Álvarez

Email: jalvarez@androgen.es.

(Trabajo recibido el 31 de agosto 2005) 